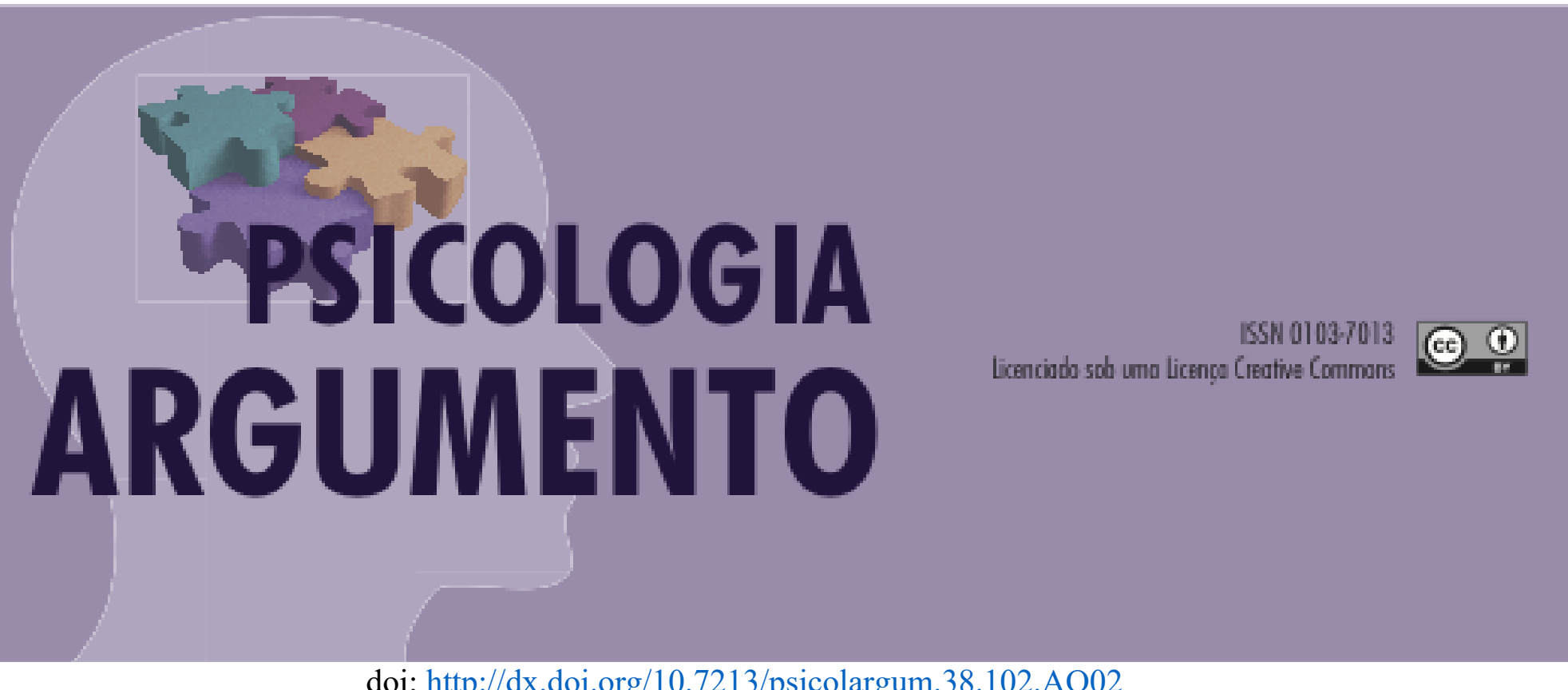

doi: $\underline{\text { http://dx.doi.org/10.7213/psicolargum.38.102.AO02 }}$

\title{
Adesão ao pós-operatório em cirurgia bariátrica: análise sistemática da literatura brasileira
}

Postoperative adherence in bariatric surgery: systematic analysis of the brazilian literature

Adherencia a la cirugía bariátrica postoperatoria: análisis sistemática de la literatura brasileña

Sara Juliana Bulgarelli Guadanhim Gonçalves

Mestranda em Psicologia no UniCEUB, DF, sara.goncalves@cfp.org.br

http://orcid.org/0000-0002-5992-0456

Marina Kohlsdorf

Professora Titular- Curso de Psicologia (FACES) do UniCEUB, Coordenadora do Programa de

Mestrado em Psicologia(ICPD) do UniCEUB, marina.kohlsdorf@ceub.edu.br http://orcid.org/0000-0002-7029-3270

Amália Raquel Perez-Nebra

Professora Titular da Faculdade de Ciências da Educação e Saúde do UniCEUB, amalia.perez@ceub.edu.br, http://orcid.org/0000-0001-8386-1233

\section{Resumo}

Adesão aos cuidados refere-se a um comportamento consonante com a abordagem e o tipo de tratamento proposto pela equipe de saúde. O presente artigo tem como objetivo realizar uma revisão sistemática das publicações entre 2009 e 2018, visando a tipificação de eixos da adesão aos cuidados após a cirurgia bariátrica e a verificação de eixos pouco ou nada explorados. Foi realizada busca bibliográfica nas bases de dados: Scielo, Lilacs, Bireme/BVS, Pepsic e Psycinfo, além de busca sistemática em revistas nacionais indexadas no Portal Capes e busca no Google Scholar e rede de Internet, incluindo-se a busca em teses e dissertações. Foram analisados 27 
trabalhos e os resultados indicaram aumento dos trabalhos publicados nos dois últimos anos, predominância de relatos de pesquisa caracterizados por metodologia transversal, delineamento descritivo/exploratório, uso de análise quantitativa, aplicação de questionário sociodemográfico e análise dos prontuários dos pacientes. As amostras pesquisadas incluíram em sua totalidade os pacientes submetidos à cirurgia bariátrica. A adesão nutricional foi mais verificada $(n=23)$, seguida da adesão ao acompanhamento psicológico $(n=11)$. Dessa forma, destaca-se a importância de mais estudos em caráter qualitativo e que focalizem temas pouco investigados, que possam contribuir de forma relevante para a compreensão sobre quais elementos estão funcionalmente envolvidos na adesão aos cuidados após a realização da cirurgia bariátrica.

Palavras-chave: obesidade; cirurgia bariátrica; adesão aos cuidados pós-operatórios, aderência.

\begin{abstract}
Adherence to care refers to behavior consistent with the approach and the type of treatment proposed by the health team. This article aims to carry out a systematic review of publications between 2009 and 2018, aiming at the typification of axes of adherence to care after bariatric surgery and the verification of axes little or nothing explored. Bibliographic search was performed in the databases: Scielo, Lilacs, Bireme / BVS, Pepsic and Psycinfo, in addition to systematic search in national magazines indexed on the Capes Portal and search on Google Scholar and Internet network, including the search in theses and dissertations. 27 studies were analyzed and the results indicated an increase in published works in the last two years, predominance of research reports characterized by cross-sectional methodology, descriptive / exploratory design, use of quantitative analysis, application of sociodemographic questionnaire and analysis of patient records. The researched samples included all patients submitted to bariatric surgery. Nutritional adherence was more verified $(n=23)$, followed by adherence to psychological monitoring $(n=11)$. Thus, the importance of more qualitative studies that focus on little-researched topics that can contribute in a relevant way to the understanding of what elements are functionally involved in adherence to care after bariatric surgery is highlighted.
\end{abstract}

Keywords: obesity; bariatric surgery; adherence to postoperative care.

\title{
Resumen
}

La adherencia a la atención se refiere al comportamiento consistente con el enfoque y el tipo de tratamiento propuesto por el equipo de salud. Este artículo tiene como objetivo llevar a cabo una revisión sistemática de publicaciones entre 2009 y 2018, con el objetivo de tipificar los ejes de adherencia a la atención después de la cirugía bariátrica y la verificación de los ejes explorados poco o nada. La búsqueda bibliográfica se realizó en las bases de datos: Scielo, Lilacs, Bireme I BVS, Pepsic y Psycinfo, además de la búsqueda sistemática en revistas nacionales indexadas en el Portal de Capes y la búsqueda en Google Scholar y la red de Internet, incluida la búsqueda en tesis y disertaciones. Se analizaron 27 estudios y los resultados indicaron un aumento en los trabajos publicados en los últimos dos años, predominio de informes de investigación caracterizados por metodología transversal, diseño descriptivo / exploratorio, uso de análisis cuantitativo, aplicación de cuestionario sociodemográfico y análisis de registros de pacientes. Las muestras investigadas incluyeron a todos los pacientes sometidos a cirugía bariátrica. La adherencia nutricional fue más verificada $(n=23)$, seguida de la adherencia al monitoreo psicológico $(n=11)$. Por lo tanto, se destaca la importancia de más estudios cualitativos que se centren en temas mal investigados, que pueden contribuir de manera relevante a la comprensión de qué elementos están involucrados funcionalmente en el cumplimiento de la atención después de realizar una cirugía bariátrica.

Palabras clave: obesidad; cirugía bariátrica; adherencia a la atención postoperatoria; adherencia.

\section{Introdução}


A obesidade é uma doença crônica global de incidência crescente e que se tornou nas três últimas décadas um dos maiores problemas de saúde pública das sociedades urbanas. Em 1999 o Ministério da Saúde reconheceu o tratamento cirúrgico das obesidades grau II, com comorbidades, e grau III segundo a classificação da Organização Mundial da Saúde (OMS) como uma alternativa eficaz para o controle dessa doença. Por ser uma cirurgia de grande porte com risco de complicações em longo prazo, preconiza-se o acompanhamento contínuo dos indivíduos operados por equipe multidisciplinar. A prevalência do comportamento de adesão ao seguimento pós-operatório tem sido considerada um importante critério para avaliação do sucesso do procedimento e a recidiva ou não da obesidade. O comparecimento escasso ou irregular dos indivíduos às consultas agendadas no período pós-operatório pode ser caracterizado por diversos fatores: a) serviços não estruturados para esse seguimento; b) dificuldade de adesão ao tratamento comum em doenças crônicas; c) grande distância entre o local de trabalho ou da residência e o local onde ocorrem as consultas; d) problemas financeiros (Scabim, 2011).

O presente artigo tem como objetivo realizar uma revisão sistemática de literatura brasileira publicada entre 2009 e 2018, visando à tipificação de eixos da adesão aos cuidados após a cirurgia bariátrica e a verificação de eixos pouco ou nada explorados. Serão apontados conceitos referentes à obesidade, cirurgia bariátrica e adesão aos cuidados pós-operatórios em cirurgia bariátrica. Também elementos abordados em revisões tradicionais de literatura, tais como: os temas focalizados, amostra, delineamentos metodológicos e tipos de análise para ilustrar de modo sistemático as principais características da literatura recente. Serão discutidos os dados relevantes dos artigos buscados na base de dados.

Adesão ao tratamento refere-se a um comportamento consonante com a abordagem e o tipo de tratamento proposto pela equipe de saúde (Gusmão \& Mion, 2006). Segundo a OMS (2016) o conceito de adesão foi proposto como a extensão com a qual o comportamento de uma pessoa, tomando medicação, seguindo uma dieta e/ou executando mudanças no estilo de vida corresponde às orientações que foram recomendadas em comum acordo com a equipe de saúde. Destaca-se pelo comprometimento do próprio paciente, sua família e equipe de saúde para que o processo de emagrecimento acarretado pela cirurgia bariátrica seja efetivo. 


\section{Obesidade e a Cirurgia Bariátrica}

A obesidade é definida pela Organização Mundial da Saúde (OMS) como um acúmulo anormal ou excessivo de gordura corporal que pode atingir graus capazes de afetar a saúde. O Índice de Massa Corporal (IMC) é uma razão simples entre o peso e a altura elevada ao quadrado, que é frequentemente usada para classificar a obesidade em adultos. A OMS estima que pelo menos 1 bilhão de pessoas apresente excesso de peso, das quais 300 milhões são obesas (OMS, 2016). Assim, considera-se obesidade quando o IMC se encontra acima de $30 \mathrm{~kg} / \mathrm{m}^{2}$ e sua gravidade pode ser definida grau I quando o IMC situa-se entre 30 e $34,9 \mathrm{~kg} / \mathrm{m}^{2}$, obesidade grau II quando IMC está entre 35 e $39,9 \mathrm{~kg} / \mathrm{m}^{2}$ e obesidade grau III quando o IMC ultrapassa $40 \mathrm{~kg} / \mathrm{m}^{2}$, que pode ser chamada também de obesidade mórbida (OMS, 2016). Estima-se que de 2\% a 8\% dos gastos em tratamentos de saúde em vários países do mundo sejam destinados à obesidade.

Sendo a obesidade uma condição médica crônica de etiologia multifatorial o seu tratamento envolve várias abordagens: nutricional, uso de medicamentos antiobesidade e prática de exercícios físicos (Fandiño, Benchimol, Coutinho \& Appolinário, 2004). Considerando as dificuldades das pessoas de seguirem o tratamento habitual e terem sucesso foi desenvolvido um tratamento via intervenção cirúrgica, a cirurgia bariátrica, que é considerada atualmente mais eficaz na perda de peso corporal (Garrido, 2004). Existem diversas técnicas de realização da cirurgia bariátrica: bypass gástrico (gastroplastia com desvio intestinal em "Y de Roux"), gastrectomia vertical, duodenal switch, banda gástrica ajustável, balão gástrico (SBCBM, 2018).

A cirurgia bariátrica resulta em uma perda de peso significativa e melhoria das comorbidades e da qualidade de vida do indivíduo. A indicação desta intervenção vem crescendo nos dias atuais e se baseia numa análise abrangente de múltiplos aspectos do paciente, aspectos físicos, psicológicos, sociais, emocionais (Garrido, 2004). Apesar de ser realizada em obesos grau II e com comorbidades, é indicada, principalmente, entre aqueles pacientes chamados de obesos mórbidos os quais são pouco responsivos ao tratamento convencional baseado na mudança nutricional e nos hábitos de vida, ou, mesmo, a medicamentos (Guerra, 2014). 
Com a realização da cirurgia é necessário se atentar para a recidiva da obesidade e ter em mente as taxas de sucesso da operação. Dessa forma, Eduardo, et al. (2017) apontaram que a perda de seguimento com as equipes de saúde além de ser uma realidade no pós-operatório está envolvida no reganho de peso por impactar negativamente no autocuidado e automonitoramento, dificultar a realização e manutenção das mudanças de hábitos exigidos pela cirurgia, além da possibilidade de detecção precoce de ganho ponderal. Por se tratar da última opção terapêutica na linha de cuidado da obesidade os fatores preditivos para a ocorrência de reganho de peso devem ser investigados e trabalhados com os pacientes.

Dados da Sociedade Brasileira de Cirurgia Bariátrica e Metabólica (SBCBM, 2018) indicam que a cirurgia bariátrica apresenta uma taxa de sucesso consistente promovendo redução do excesso de peso e diminuição das comorbidades. A taxa de sucesso foi estimada em $90 \%$ dos pacientes que aliaram a cirurgia bariátrica ao papel fundamental do preparo pré-operatório e acompanhamento multidisciplinar pósoperatório no resultado final da cirurgia. Apontaram ainda que problemas relacionados a recidiva da obesidade podem ocorrer em cerca de $10 \%$ a $15 \%$ dos casos. E que o reganho de aproximadamente $10 \%$ do total de peso perdido após a cirurgia bariátrica é considerado normal a partir de 24 meses de realizada a cirurgia e que não é considerado como recidiva da obesidade.

\section{Adesão aos Cuidados Pós-operatórios em Cirurgia Bariátrica}

A cirurgia bariátrica requer modificações significativas no estilo de vida, seja pela mudança no comportamento alimentar, seja pela introdução da suplementação, introdução do exercício físico ou pelo longo acompanhamento multidisciplinar póscirúrgico, sendo essencial um grande esforço e adesão terapêutica pós-operatória para que o processo resulte em sucesso em longo prazo (Teixeira \& Maia, 2011).

Etimologicamente o termo adesão significa obediência, fidelidade, aprovação, solidariedade a uma ideia ou causa (Ferreira, 1999). Na literatura médica uma das definições de adesão mais divulgadas foi formulada por Haynes, em 1979, na qual é enfatizada a extensão com a qual o comportamento de uma pessoa, o paciente ou seu cuidador, coincide com a orientação do profissional (Garrido, 2004). 
A OMS (2003) reconheceu que a adesão aos cuidados compreende um conjunto de ações que podem incluir tomar medicamentos, obter imunização, comparecer ao agendamento de consultas e adotar hábitos saudáveis de vida, como: reeducação alimentar, práticas de atividade física regular, prevenção ao consumo de álcool e tabaco. E que tais ações podem ser entendidas como ações de autocontrole e autorregulação.

Ainda segundo a OMS o fenômeno da adesão é determinado pela interação de cinco dimensões: a) fatores relacionados ao paciente: percepção da seriedade do problema, desconhecimento, experiência com a doença no contexto familiar, suas crenças, hábitos de vida, autoestima, percepção de autoeficácia, sobrecarga de necessidade de controle; b) fatores socioeconômicos: sexo, idade, etnia, estado civil, escolaridade e nível socioeconômico; c) sistema e equipe de saúde: política de saúde, acesso ao serviço de saúde, tempo de espera versus tempo de atendimento, manejo da equipe de saúde; d) fatores relacionados aos cuidados: custo, efeitos indesejáveis, esquemas terapêuticos complexos; e) fatores relacionados à doença: cronicidade, ausência de sintomas e consequências tardias (OMS, 2003).

Consequentemente a adesão aos cuidados expressa a compreensão e a cooperação do paciente em face do planejamento e da implantação dos próprios cuidados, o que pressupõe comportamento ativo por parte de quem adere e, dessa forma, o processo de avaliação da adesão aos cuidados pós-operatórios torna-se bastante complexo.

A não adesão aos cuidados, a dificuldade de o paciente usar a medicação prescrita, de seguir a dieta ou de modificar seu estilo de vida de acordo com as orientações da equipe multidisciplinar é considerada um problema pelos profissionais de saúde pois acarreta o reganho de peso e pode trazer complicações na saúde dos pacientes (Camargo-Borges \& Japur, 2008).

\section{Objetivos}

O presente artigo tem como objetivo realizar uma revisão sistemática de literatura brasileira publicada entre 2009 e 2018, visando a tipificação de eixos da adesão aos cuidados após a cirurgia bariátrica e a verificação de eixos pouco ou nada explorados.

\section{Método}




\section{Procedimento para coleta de dados}

Foi realizado levantamento bibliográfico a partir da busca eletrônica de trabalhos nacionais disponibilizados nas bases de dados: Scielo (Scientific Eletronic Library Online), Lilacs (Literatura Latino-americana de Ciências da Saúde), Bireme/BVS (Biblioteca Virtual em Saúde) e Pepsic, além de busca sistemática em revistas nacionais indexadas no Portal Capes (Coordenação e Aperfeiçoamento de Pessoal de Nível Superior) e busca no Google Scholar e rede de Internet, incluindo-se a busca em teses e dissertações. As palavras-chave utilizadas para as buscas foram adesão, aderência, cuidados pós-operatórios, cirurgia bariátrica, obesidade. Inicialmente, foram combinadas as palavras adesão e cuidados pós-operatórios e cirurgia bariátrica e obesidade. Combinados também aderência e cuidados pós-operatórios e cirurgia bariátrica e obesidade. Posteriormente as palavras-chave foram pesquisadas individualmente. As buscas foram realizadas na língua portuguesa. Os critérios para inclusão de dados foram: (a) trabalhos publicados entre 2009 e 2018, para que se faça menção a literatura mas recente sobre a temática; (b) modalidade de produção (artigos de pesquisa, estudos de caso, revisões de literatura, dissertações e teses); e (c) temática sobre adesão aos cuidados após a cirurgia bariátrica.

\section{Resultados}

Os trabalhos foram filtrados nas plataformas das bases de dados. Após o filtro inicial, ou seja, a inserção da combinação das palavras-chave nas plataformas, foram selecionados 2.346 trabalhos que referiram sobre adesão em seu conteúdo. O segundo filtro foi realizado visando retirar os trabalhos duplicados, restando 1.184 trabalhos. Após foi realizada a busca individual das palavras-chave, restando 323 trabalhos. Posteriormente foram excluídos os trabalhos anteriores ao ano de 2009 restando 189. Em seguida foram lidos os títulos e resumos dos referidos trabalhos de forma a ser verificado o tema adesão aos cuidados pós-operatórios em cirurgia bariátrica, restando 74 trabalhos. Posteriormente os trabalhos com o tema adesão foram lidos por completo restando amostra de 27 trabalhos. Conforme Figura 1.

Figura 1- Mapeamento das filtragens de verificação dos trabalhos 


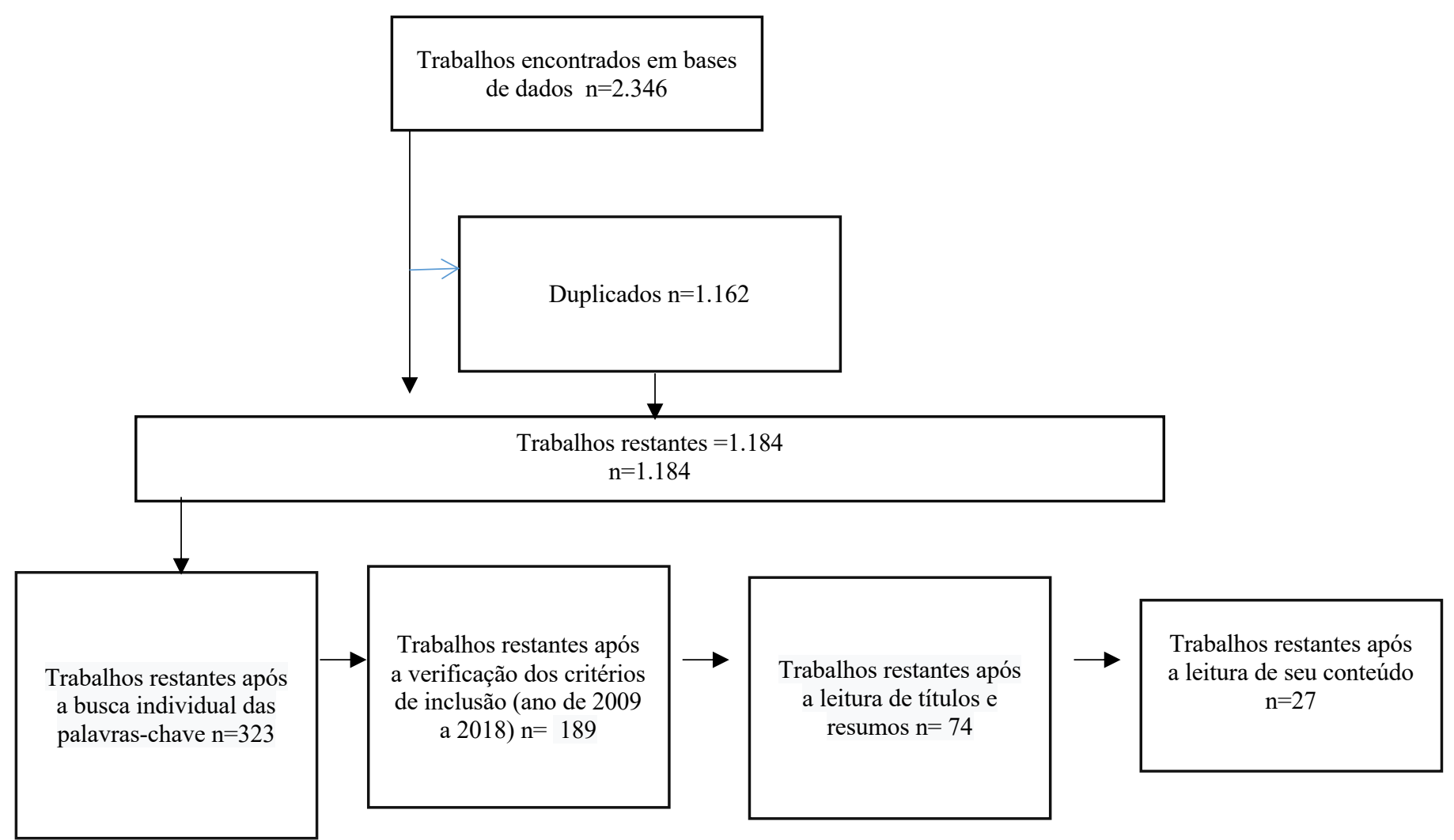

Foram localizados 27 trabalhos publicados, sendo cinco revisões de literatura, um estudo de caso e 21 relatos de pesquisa empírica.

Figura 2. Indicação dos trabalhos por ano

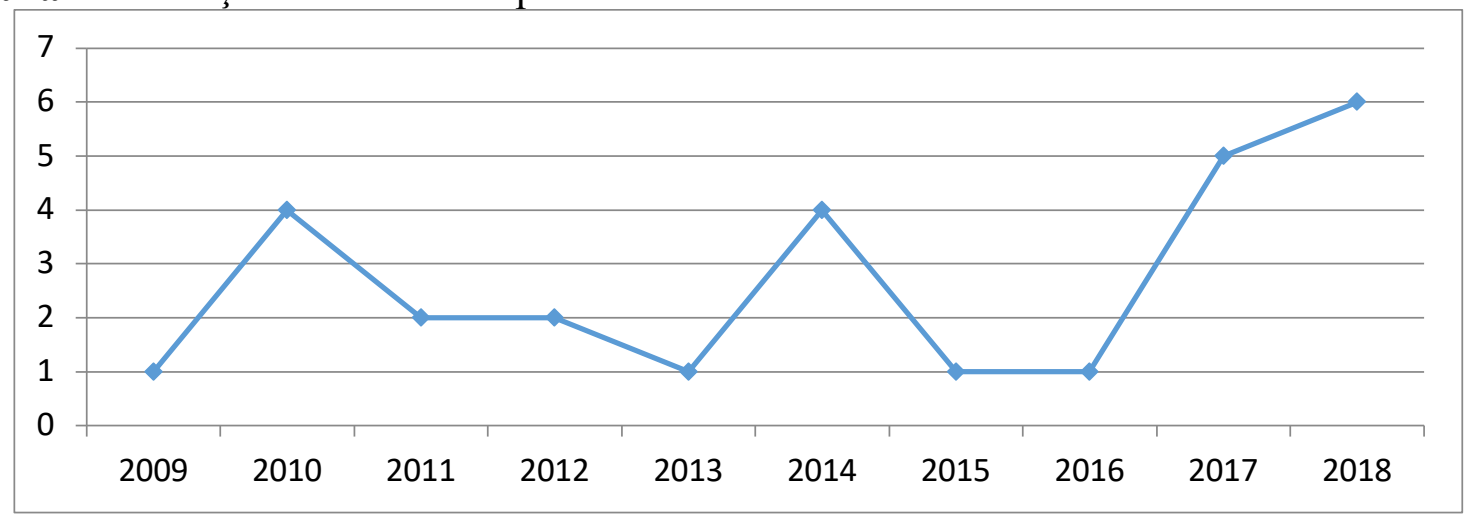

A Figura 2 mostra que os anos de 2017 e de 2018 registraram um aumento no número de publicações.

Os trabalhos foram categorizados segundo ano, tema principal, tipo de análise (quantitativa, qualitativa, mista), tipologia de adesão (nutricional, psicológico, acompanhamento multidisciplinar, consulta com cirurgião, atividade física, 
suplementação), tipo de pesquisa (revisão de literatura, relato de pesquisa empírica ou estudo de caso) e amostra. Conforme Tabela 1.

Tabela 1- Demonstrativo do ano e tema principal e sua correspondência com a análise, a tipologia da adesão, tipo de pesquisa realizado e a amostra

\begin{tabular}{|c|c|c|c|c|c|}
\hline Ano & Tema Principal & Análise & Tipologia de Adesão & Tipo de Pesquisa & Amostra \\
\hline 2009 & $\begin{array}{l}\text { Um novo olhar sobre a } \\
\text { cirurgia bariátrica e os } \\
\text { transtornos alimentares }\end{array}$ & Qualitativa & $\begin{array}{l}\text { Psicológico, } \\
\text { nutricional }\end{array}$ & Estudo de Caso & 5 pacientes \\
\hline 2010 & $\begin{array}{l}\text { Fatores envolvidos na } \\
\text { adesão ao tratamento } \\
\text { multidisciplinar após a } \\
\text { cirurgia bariátrica }\end{array}$ & $\begin{array}{l}\text { Quantita } \\
\text { tiva }\end{array}$ & $\begin{array}{l}\text { Psicológico, } \\
\text { nutricional, } \\
\text { acompanhamen } \\
\text { to multidisciplinar }\end{array}$ & $\begin{array}{l}\text { Relato pesquisa } \\
\text { empírica }\end{array}$ & 87 pacientes \\
\hline 2010 & $\begin{array}{l}\text { Adesão ao tratamento } \\
\text { multidisciplinar } 6 \\
\text { meses após a cirurgia } \\
\text { bariátrica }\end{array}$ & $\begin{array}{l}\text { Quantita } \\
\text { tiva }\end{array}$ & $\begin{array}{l}\text { Psicológico, } \\
\text { nutricional, } \\
\text { consulta com } \\
\text { cirurgião, } \\
\text { acompanhamen to } \\
\text { multidiscipli } \\
\quad \text { nar }\end{array}$ & $\begin{array}{l}\text { Rev. de } \\
\text { Literatura }\end{array}$ & 17 trabalhos \\
\hline 2010 & $\begin{array}{l}\text { Acompanhamento } \\
\text { psicológico tardio em } \\
\text { pacientes submetidos } \\
\text { à cirurgia bariátrica }\end{array}$ & $\begin{array}{l}\text { Quantita } \\
\text { tiva }\end{array}$ & $\begin{array}{l}\text { Nutricional, } \\
\text { suplementação, } \\
\text { psicológico }\end{array}$ & $\begin{array}{l}\text { Relato } \\
\text { pesquisa } \\
\text { empírica }\end{array}$ & 46 pacientes \\
\hline 2010 & $\begin{array}{l}\text { Aspectos da aderência } \\
\text { após cirurgia bariátrica }\end{array}$ & Mista & $\begin{array}{l}\text { Nutricional, } \\
\text { suplementa ção, } \\
\text { atividade física, } \\
\text { acompanha } \\
\text { mento } \\
\text { multidisciplinar } \\
\text {,psicológico }\end{array}$ & $\begin{array}{c}\text { Relato pesquisa } \\
\text { empírica }\end{array}$ & 15 pacientes \\
\hline
\end{tabular}

\begin{tabular}{|c|c|c|c|c|c|}
\hline$\overline{2011}$ & $\begin{array}{l}\text { Adesão ao seguimento } \\
\text { nutricional } \\
\text { ambulatorial pós- } \\
\text { operatório de cirurgia } \\
\text { bariátrica }\end{array}$ & $\begin{array}{l}\text { Quantita } \\
\text { tiva }\end{array}$ & Nutricional & $\begin{array}{l}\text { Relato } \\
\text { pesquisa } \\
\text { empírica }\end{array}$ & $\begin{array}{l}241 \\
\text { pacientes }\end{array}$ \\
\hline$\overline{2011}$ & $\begin{array}{l}\text { Fatores de insucesso } \\
\text { na cirurgia bariátrica }\end{array}$ & $\begin{array}{l}\text { Quantita } \\
\text { tiva }\end{array}$ & $\begin{array}{l}\text { Nutricional, } \\
\text { psicológico }\end{array}$ & $\begin{array}{l}\text { Rev. de } \\
\text { Literatura }\end{array}$ & 23 trabalhos \\
\hline 2012 & $\begin{array}{l}\text { Cirurgia bariátrica e } \\
\text { melhora na qualidade } \\
\text { de vida }\end{array}$ & $\begin{array}{l}\text { Quantita } \\
\text { tiva }\end{array}$ & Nutricional & $\begin{array}{l}\text { Rev. de } \\
\text { Literatura }\end{array}$ & 4 trabalhos \\
\hline 2012 & $\begin{array}{l}\text { Comportamento } \\
\text { alimentar e imagem } \\
\text { corporal em mulheres } \\
\text { submetidas a cirurgia } \\
\text { bariátrica }\end{array}$ & $\begin{array}{l}\text { Quantita } \\
\text { tiva }\end{array}$ & $\begin{array}{l}\text { Nutricional, } \\
\text { psicológico, } \\
\text { atividade física }\end{array}$ & $\begin{array}{l}\text { Relato } \\
\text { pesquisa } \\
\text { empírica }\end{array}$ & 50 pacientes \\
\hline 2013 & $\begin{array}{l}\text { Avaliação da } \\
\text { frequência em } \\
\text { consultas nutricionais } \\
\text { dos pacientes após a } \\
\text { cirurgia bariátrica }\end{array}$ & $\begin{array}{l}\text { Quantita } \\
\text { tiva }\end{array}$ & Nutricional & $\begin{array}{l}\text { Relato } \\
\text { pesquisa } \\
\text { empírica }\end{array}$ & 469 pacientes \\
\hline
\end{tabular}




\begin{tabular}{|c|c|c|c|c|c|}
\hline 2014 & $\begin{array}{l}\text { A importância do } \\
\text { acompanhamento } \\
\text { nutricional e } \\
\text { psicológico no pré e } \\
\text { pós-operatório da } \\
\text { cirurgia bariátrica }\end{array}$ & $\begin{array}{l}\text { Quantita } \\
\text { tiva }\end{array}$ & $\begin{array}{l}\text { Nutricional, } \\
\text { psicológico }\end{array}$ & $\begin{array}{l}\text { Relato } \\
\text { pesquisa } \\
\text { empírica }\end{array}$ & 25 pacientes \\
\hline 2014 & $\begin{array}{l}\text { Qualidade e } \\
\text { comportamento } \\
\text { alimentar após a } \\
\text { cirurgia bariátrica }\end{array}$ & $\begin{array}{l}\text { Quantita } \\
\text { tiva }\end{array}$ & Nutricional & $\begin{array}{l}\text { Relato } \\
\text { pesquisa } \\
\text { empírica }\end{array}$ & 95 pacientes \\
\hline 2014 & $\begin{array}{l}\text { Avaliação das práticas } \\
\text { de atividade física em } \\
\text { pacientes que } \\
\text { realizaram a cirurgia } \\
\text { bariátrica }\end{array}$ & $\begin{array}{l}\text { Quantita } \\
\text { tiva }\end{array}$ & $\begin{array}{l}\text { Atividade } \\
\text { física }\end{array}$ & $\begin{array}{l}\text { Relato } \\
\text { pesquisa } \\
\text { empírica }\end{array}$ & 11 pacientes \\
\hline 2014 & $\begin{array}{l}\text { Apoio social e reganho } \\
\text { de peso }\end{array}$ & Quantitativa & $\begin{array}{l}\text { Nutricional, } \\
\text { atividade } \\
\text { física, } \\
\text { suplementa ção } \\
\end{array}$ & $\begin{array}{l}\text { Relato } \\
\text { pesquisa } \\
\text { empírica }\end{array}$ & $\begin{array}{l}6 \text { pacientes } \\
\text { e } 3 \\
\text { cuidadores }\end{array}$ \\
\hline 2015 & $\begin{array}{l}\text { Associação entre } \\
\text { fatores psicossociais e } \\
\text { aderência ao } \\
\text { tratamento de } \\
\text { pacientes submetidos } \\
\text { a cirurgia bariátrica }\end{array}$ & $\begin{array}{l}\text { Quantita } \\
\text { tiva }\end{array}$ & $\begin{array}{l}\text { Nutricional, } \\
\text { atividade física, } \\
\text { acompanhamen } \\
\text { to multidiscipli } \\
\text { nar, psicológico }\end{array}$ & $\begin{array}{l}\text { Relato } \\
\text { pesquisa } \\
\text { empírica }\end{array}$ & 39 pacientes \\
\hline 2016 & $\begin{array}{l}\text { Variáveis psicológicas } \\
\text { associadas à cirurgia } \\
\text { bariátrica }\end{array}$ & Qualitativa & Psicológico & $\begin{array}{l}\text { Rev. De } \\
\text { Literatura }\end{array}$ & 6 trabalhos \\
\hline 2017 & $\begin{array}{l}\text { A importância do } \\
\text { acompanhamento } \\
\text { nutricional em } \\
\text { pacientes que } \\
\text { realizaram a cirurgia } \\
\text { bariátrica }\end{array}$ & $\begin{array}{l}\text { Quantita } \\
\text { tiva }\end{array}$ & Nutricional & $\begin{array}{l}\text { Relato } \\
\text { pesquisa } \\
\text { empírica }\end{array}$ & 43 pacientes \\
\hline 2017 & $\begin{array}{l}\text { Práticas Alimentares } \\
\text { de pacientes após a } \\
\text { cirurgia bariátrica }\end{array}$ & $\begin{array}{l}\text { Qualitativ } \\
\text { a }\end{array}$ & Nutricional & $\begin{array}{l}\text { Rev. De } \\
\text { Literatura }\end{array}$ & 13 trabalhos \\
\hline 2017 & $\begin{array}{l}\text { Avaliação da redução } \\
\text { de excesso de peso e } \\
\text { de carências } \\
\text { nutricionais em } \\
\text { pacientes bariátricos }\end{array}$ & $\begin{array}{l}\text { Quantita } \\
\text { tiva }\end{array}$ & Nutricional & $\begin{array}{l}\text { Relato } \\
\text { pesquisa } \\
\text { empírica }\end{array}$ & 24 pacientes \\
\hline 2017 & $\begin{array}{l}\text { Cirurgia bariátrica no } \\
\text { pós-operatório tardio e } \\
\text { a adesão ao tratamento } \\
\text { nutricional }\end{array}$ & $\begin{array}{l}\text { Quantita } \\
\text { tiva }\end{array}$ & Nutricional & $\begin{array}{l}\text { Relato } \\
\text { pesquisa } \\
\text { empírica }\end{array}$ & 30 pacientes \\
\hline 2017 & $\begin{array}{l}\text { Cirurgia bariátrica: a } \\
\text { percepção do paciente } \\
\text { frente ao impacto físico, } \\
\text { psicológico e social }\end{array}$ & $\begin{array}{l}\text { Qualita } \\
\text { tiva }\end{array}$ & $\begin{array}{l}\text { Nutricional, } \\
\text { acompanha } \\
\text { mento } \\
\text { multidisciplinar } \\
\text {, psicológico }\end{array}$ & $\begin{array}{l}\text { Relato } \\
\text { pesquisa } \\
\text { empírica }\end{array}$ & 15 pacientes \\
\hline 2018 & $\begin{array}{l}\text { Avaliação da condição } \\
\text { de saúde e da } \\
\text { qualidade de vida após } \\
\text { a cirurgia bariátrica }\end{array}$ & $\begin{array}{l}\text { Quantita } \\
\text { tiva }\end{array}$ & $\begin{array}{l}\text { Atividade física, } \\
\text { acompanhamen } \\
\text { to multidisci } \\
\text { plinar }\end{array}$ & $\begin{array}{l}\text { Relato } \\
\text { pesquisa } \\
\text { empírica }\end{array}$ & 59 pacientes \\
\hline
\end{tabular}




\begin{tabular}{|c|c|c|c|c|c|}
\hline 2018 & $\begin{array}{l}\text { Avaliação da } \\
\text { qualidade de vida, } \\
\text { perda de peso e } \\
\text { comorbidades de } \\
\text { pacientes submetidos } \\
\text { a cirurgia bariátrica }\end{array}$ & $\begin{array}{l}\text { Quantita } \\
\text { tiva }\end{array}$ & $\begin{array}{l}\text { Nutricional, } \\
\text { psicológico }\end{array}$ & $\begin{array}{l}\text { Relato } \\
\text { pesquisa } \\
\text { empírica }\end{array}$ & $\begin{array}{l}103 \\
\text { pacientes }\end{array}$ \\
\hline 2018 & $\begin{array}{l}\text { Comportamento e } \\
\text { hábitos alimentares } \\
\text { dos pacientes pós- } \\
\text { cirurgia bariátrica }\end{array}$ & $\begin{array}{l}\text { Quantita } \\
\text { tiva }\end{array}$ & Nutricional & $\begin{array}{l}\text { Relato } \\
\text { pesquisa } \\
\text { empírica }\end{array}$ & $\begin{array}{l}100 \\
\text { pacientes }\end{array}$ \\
\hline 2018 & $\begin{array}{l}\text { Fatores preditivos da } \\
\text { perda de seguimento } \\
\text { de pacientes } \\
\text { submetidos à cirurgia } \\
\text { bariátrica }\end{array}$ & $\begin{array}{l}\text { Quantita } \\
\text { tiva }\end{array}$ & $\begin{array}{l}\text { Nutricional, } \\
\text { acompanhamen } \\
\text { to multidisci } \\
\text { plinar }\end{array}$ & $\begin{array}{l}\text { Relato } \\
\text { pesquisa } \\
\text { empírica }\end{array}$ & $\begin{array}{l}559 \\
\text { pacientes }\end{array}$ \\
\hline 2018 & $\begin{array}{l}\text { Mudanças de hábitos } \\
\text { de vida de pacientes } \\
\text { em pós-operatório da } \\
\text { cirurgia bariátrica }\end{array}$ & $\begin{array}{l}\text { Quantita } \\
\text { tiva }\end{array}$ & $\begin{array}{l}\text { Nutricional, } \\
\text { atividade física }\end{array}$ & $\begin{array}{l}\text { Relato } \\
\text { pesquisa } \\
\text { empírica }\end{array}$ & 92 pacientes \\
\hline 2018 & $\begin{array}{l}\text { Percepção de risco da } \\
\text { cirurgia bariátrica em } \\
\text { pacientes com } \\
\text { diferentes } \\
\text { comorbidades } \\
\text { associadas à obesidade }\end{array}$ & $\begin{array}{l}\text { Quantita } \\
\text { tiva }\end{array}$ & $\begin{array}{l}\text { Acompanhamento } \\
\text { multidis } \\
\text { ciplinar }\end{array}$ & $\begin{array}{l}\text { Relato } \\
\text { pesquisa } \\
\text { empírica }\end{array}$ & $\begin{array}{l}128 \\
\text { pacientes }\end{array}$ \\
\hline
\end{tabular}

A Tabela 1 mostra que as publicações descritas como relato de pesquisa empírica apresentaram $n=21$, enquanto que as revisões de literatura apresentaram $n=5$ e houve um estudo de caso analisado, o que pode ser indicativo da necessidade de os pesquisadores e estudiosos do assunto de mais conhecimento sobre o assunto. Há predominância da análise quantitativa $\operatorname{com} n=22$, seguida da análise qualitativa $\operatorname{com} n=$ 4 e um estudo indicou a análise mista dos dados.

Os trabalhos analisados indicam a grande predominância de realização da cirurgia por mulheres, o que pode indicar maior preocupação deste sexo com a aparência e estética. Indicam também, mesmo que indiretamente, ou seja, não esteja explícito o texto, a questão de melhora da qualidade de vida após a realização da cirurgia bariátrica. Pesquisa realizada no Brasil pelo Ministério da Saúde em 2008 apontou que o crescimento da obesidade foi maior entre os brasileiros adultos de 25 a 34 anos e 35 a 44 anos, com 84,2\% e 81,1\%, respectivamente. Apesar de o excesso de peso ser mais comum entre os homens, em 2018, as mulheres apresentaram obesidade ligeiramente maior, com 20,7\%, em relação aos homens, 18,7\%. A pesquisa registrou também que mais da metade da população, 55,7\% tem excesso de peso (MS, 2008). Tais dados podem ser indicativo de que mais mulheres realizam a cirurgia bariátrica pois mais mulheres são obesas e cuidam mais da saúde do que os homens (SBCBM, 2018). 
A adesão aos cuidados pós-operatórios, no que se refere à frequência em consultas multidisciplinares, em sete dos trabalhos analisados, teve relação quanto a quanto à renda familiar, demonstrando que quanto menor a renda familiar, menor a adesão, haja vista que algumas consultas são pagas. A distância da moradia também possui relação na adesão, quanto mais perto o local de moradia, há maior a adesão.

Quanto ao apoio social, 16 das pesquisas avaliadas revelaram que quanto menor a percepção de apoio social afetivo, emocional/informacional e apoio social total por parte dos pacientes, maior foi a adesão ao tratamento médico. Apontaram também a importância do suporte familiar ao tratamento nutricional no período após a cirurgia bariátrica, sobretudo logo após o procedimento cirúrgico, quando o paciente ainda se encontra debilitado e, consequentemente, mais dependente da própria família. Cabe ressaltar que as dimensões apoio/suporte social e apoio/suporte familiar não foram categorizadas pela OMS.

Indicaram, também, que as consultas com o nutricionista favoreceram sensivelmente a adesão do paciente às consultas médicas e com o psicólogo, concluindo-se que a assistência prestada por aquele profissional favoreceu a inclusão do paciente no acompanhamento multidisciplinar efetivo. Esse dado parece revelar o nutricionista como o profissional da equipe que adotou atitudes interdisciplinares mais eficazes e/ou em maior quantidade, o que deve beneficiar o paciente na direção de melhor nível de saúde. Por fim, inferiram que a adesão aos cuidados após a cirurgia bariátrica aumentou o índice de satisfação com a autoimagem corporal e diminuiu significativamente os níveis de ansiedade, aumentou o retorno social e o desejo de mudança de vida. O que ratifica o descrito pelo eixo ou dimensão A da OMS.

Temas como adesão ao tratamento multidisciplinar após a cirurgia bariátrica, fatores de insucesso após a realização da cirurgia bariátrica, melhora na qualidade de vida após a cirurgia, importância da realização de atividade física após a cirurgia, variáveis psicológicas associadas à cirurgia bariátrica e práticas alimentares e de suplementação após o procedimento cirúrgico foram os assuntos norteadores das produções teóricas brasileiras durante o período analisado. Questões importantes como a comunicação médico-paciente, comunicação paciente com a equipe de saúde (destacados no eixo $\mathrm{C}$ da categorização da OMS) não foram destacados nos trabalhos pesquisados. Tais dados são demonstrativos de que há a necessidade de pesquisas que abordem tais assuntos. 
Ressalta-se o predomínio de estudos descrevendo a adesão ao tratamento nutricional $(n=23)$, seguido de adesão ao tratamento psicológico $(n=11)$, indicando que a adesão ao tratamento nutricional é amplamente mais realizada pelos pacientes. A adesão à atividade física $(n=7)$, suplementação $(n=3)$ podem ser tidos como a dimensão A (fatores relacionados ao paciente) da categorização realizada pela OMS. Acompanhamento multidisciplinar - consulta com endocrinologista, cardiologista e pneumologista $(n=8)$, e consulta com médico cirurgião $(\mathrm{n}=1)$, são descritos como a dimensão, ou eixo, $\mathrm{C}$ (sistema ou equipe de saúde) da OMS.

Houve grande incidência de trabalhos combinando os seguimentos de adesão $(n=15)$. A prevalência de combinações foi quanto ao comportamento nutricional, observando-se 14 trabalhos combinando o seguimento nutricional com algum ou alguns outros seguimentos, como acompanhamento multidisciplinar (eixo C da categorização da OMS), suplementação (eixo A da categorização da OMS), acompanhamento psicológico (eixo A da categorização da OMS), renda familiar (eixo B da categorização da OMS), dentre outros. A maior incidência de combinações foi quanto ao comportamento nutricional e acompanhamento psicológico $(n=11)$. Somente quatro trabalhos não indicaram o eixo nutricional em suas pesquisas.

Os autores utilizaram instrumentos para mensuração da adesão, como: Questionário sociodemográfico $(n=11)$, Prontuários $(n=5)$, Questionário BAROS (Nicareta et al., 2015) $(n=4)$, Entrevista $(n=3)$, Questionário holandês do comportamento alimentar (QHCA) (Almeida, Loureiro \& Santos, 2001) com n=2, Fichas de cadastro, Recordatório de consumo alimentar habitual e Questionário de percepção de risco (Prasad et al., 2014) com $n=1$ cada. Tais dados demonstram que não há um instrumento único que sirva para mensurar a adesão aos cuidados pós-operatórios em cirurgia bariátrica, o que caracteriza uma complexidade na sua mensuração.

Alguns pesquisadores (Ehrenbrink, Pinto \& Prando, 2009; Menegotto, Cruz, Soares, Nunes \& Branco-Filho, 2013) relataram que o processo cirúrgico demanda uma intensa adesão dos pacientes no período pós-operatório, pois implica modificações de hábitos alimentares, comportamentais e de estilo de vida. Rocha \& Hociko (2018) verificaram que dos que reganharam peso, $81,69 \%$ não fazem acompanhamento nutricional atualmente.

Medeiros (2017) e Soares, et al. (2017), confirmaram que o acompanhamento nutricional no pós-operatório da cirurgia bariátrica pode favorecer a identificação e a 
intervenção precoce na perda de peso insuficiente ou na recidiva de peso, assim como em deficiências nutricionais, contribuindo favoravelmente para o sucesso do tratamento cirúrgico.

Marchesini (2010) destacou que a maioria dos pacientes bariátricos que se sentiu motivada a realizar a cirurgia é calcada nos retornos sociais, nos padrões estéticos (estas duas não dimensionadas pela OMS) e no desejo psicológico de mudança de vida (eixo A da OMS). A importância do acompanhamento psicológico foi também ressaltada por Fagundes et al. (2016), Eduardo, et al. (2017) e Castanha, et al. (2018) indicando a necessidade de um acompanhamento multiprofissional na melhoria da autoestima, o que possibilita a ressignificação desse novo corpo, trabalhando, por exemplo, alterações na percepção da imagem corporal e assim propiciando uma melhora nos relacionamentos sociais e no interesse sexual.

Ressalta-se que nenhum estudo nesta amostra foi caracterizado como longitudinal e que estes estudos são fundamentais neste contexto, considerando que a adesão aos cuidados pós-operatórios em cirurgia bariátrica envolve uma gama de procedimentos e de profissionais e se estendem ao longo de toda a vida. Os trabalhos foram caracterizados por metodologia transversal, delineamento descritivo/exploratório.

\section{Discussão}

De acordo com o objetivo deste artigo, foram encontrados os eixos nutricional, acompanhamento psicológico, acompanhamento multidisciplinar, realização de atividade física, suplementação e acompanhamento com médico cirurgião.

Os temas abordados pelos estudos focalizaram principalmente a questão nutricional após a realização da cirurgia bariátrica, o que denota a preocupação dos pacientes nesse seguimento principalmente nos primeiros 12 meses após a realização da cirurgia. $\mathrm{O}$ acompanhamento nutricional no pós-operatório da cirurgia bariátrica pode favorecer a identificação e a intervenção precoce na perda de peso insuficiente ou na recidiva de peso, assim como em deficiências nutricionais, contribuindo favoravelmente para o sucesso do tratamento cirúrgico (Fernandez, Gomes, Sá, Marcuartú, \& Sousa, 2017).

A análise dos dados apresentados depreende-se a necessidade de acompanhamento multidisciplinar de qualidade para que os resultados almejados sejam efetivamente alcançados, de forma a garantir a melhora na qualidade de vida do paciente 
(Schakarowski, Padoin, Mottin, \& Castro, 2018). Todavia tal aspecto não é muito abordado nos trabalhos analisados, carecendo de maior abrangência em estudos futuros.

Entretanto, mesmo diante dos resultados apresentados, destaca-se a necessidade de mais estudos que investiguem a adesão aos cuidados pós-operatórios em cirurgia bariátrica, como o aspecto psíquico da adesão, relação com a comida, compulsão alimentar, descontrole de impulso alimentar, suporte social, suporte familiar, autorregulação emocional e autorregulação da ingestão alimentar. Tais estudos propiciariam ao paciente melhor entendimento de sua escolha e de suas consequências.

Cabe ressaltar também a escassez de estudos com delineamento qualitativo que possam contribuir de forma relevante para a compreensão sobre quais elementos estão funcionalmente envolvidos na adesão aos cuidados após a realização da cirurgia bariátrica. Ainda, há a escassez de estudos com delineamento experimental ou quase experimental que possam contribuir de forma relevante para a compreensão sobre quais elementos estão funcionalmente envolvidos na adesão aos cuidados após a realização da cirurgia bariátrica. Percebe-se que trabalhos que avaliam a influência de características psicossociais no tratamento de pacientes de cirurgia bariátrica são escassos na psicologia/psiquiatria. Observa-se grande número de pesquisas direcionadas para outras áreas, como a medicina, a nutrição e a enfermagem.

Destaca-se a necessidade de diversificação dos tipos de análise e instrumentos ou técnicas utilizadas, além da validação de um instrumento global que meça a adesão aos cuidados pós-operatórios em suas vertentes conforme os cinco eixos da OMS, quais sejam: a) fatores relacionados ao paciente (percepção da seriedade do problema, desconhecimento, experiência com a doença no contexto familiar, suas crenças, hábitos de vida, autoestima, percepção de autoeficácia, sobrecarga de necessidade de controle); b) fatores socioeconômicos (sexo, idade, etnia, estado civil, escolaridade e nível socioeconômico); c) sistema e equipe de saúde (política de saúde, acesso ao serviço de saúde, tempo de espera versus tempo de atendimento, manejo da equipe de saúde); d) fatores relacionados aos cuidados (custo, efeitos indesejáveis, esquemas terapêuticos complexos); e) fatores relacionados à doença (cronicidade, ausência de sintomas e consequências tardias); além de constar no instrumento eixos que abarquem o suporte social, suporte familiar, resiliência, autorregulação emocional, autorregulação na ingestão alimentar (que não foram categorizados pela OMS). 
Fica clara a ausência de estudos que contemplem o comportamento médicopaciente, comportamento paciente e equipe de saúde, o envolvimento dos cuidadores no processo de adesão; além de poucos estudos que envolvem a tomada da medicação, a necessidade de mudança no estilo de vida do próprio paciente, a funcionalidade do suporte social e familiar. Quesitos estes que deveriam constar no instrumento que avaliasse a adesão aos cuidados pós-operatórios em cirurgia bariátrica.

Dessa forma, torna-se imprescindível que pesquisadores brasileiros ampliem os estudos na área e abordem os diversos fatores neste contexto, de forma a colaborar na pesquisa sobre o tema e estabelecer um protocolo de avaliação da adesão aos cuidados pós-operatórios, além de estabelecer protocolos de intervenção e avaliação da eficácia destas intervenções.

Para Souza (2008), o desenvolvimento de diferentes técnicas para medir a adesão é o indício de que nenhuma delas é a melhor, deixando-a como uma questão difícil de mensuração. Cada método de quantificação descrito na literatura tem suas limitações e não há um consenso que possa ser tomado como padrão ouro, apesar de existirem várias estratégias. Ressalta-se que alguns métodos utilizados não abarcam a questão da adesão em si e como um todo, deixando uma lacuna que necessita ser preenchida. Daí a importância de mais estudos na área e o estabelecimento de protocolos de avaliação e de intervenção.

Gusmão e Mion (2006) afirmam que ainda existe campo aberto para pesquisa em relação à adesão. Tal afirmação pode ser explicada pelos diferentes métodos aplicados, diferentes pontos de corte, e pela ausência de um método que possa ser considerado padrão ouro e que represente as várias dimensões que envolvem o processo de adesão ao tratamento da cirurgia bariátrica.

Apesar de a cirurgia bariátrica ter sido amplamente estudada em todo o mundo, a avaliação da adesão ao tratamento ainda constitui um desafio tanto para os profissionais, quanto para as pessoas que se submeteram a tal cirurgia, merecendo ser estudada mais intensamente de forma a ser oferecida à sociedade uma alternativa de entendimento e de combate da falta de informação e da discriminação da cirurgia.

Parece necessário fortalecer a base instrumental dos profissionais da área da saúde no que concerne à adesão multidisciplinar após a cirurgia bariátrica. Esse incremento tem o objetivo de incentivar a participação ativa do indivíduo no seu processo de saúde/doença por meio de uma abordagem interdisciplinar focada na continuidade do 
tratamento da obesidade. Por conseguinte, torna-se relevante a identificação dos principais fatores que interferem na adesão, a fim de se propor intervenções sistemáticas que efetivamente viabilizem a promoção da adesão multidisciplinar do paciente, considerando as especificidades no contexto da cirurgia bariátrica.

Há uma carência de publicações e nesse sentido novos estudos devem ser realizados para verificar o sucesso da cirurgia, a curto, médio e longo prazo, monitorar a evolução dos pacientes em relação à perda de peso, aspectos alimentares e psicológicos, adesão à dieta, no sentido de promover o aconselhamento dietético adequado e o impacto na perda ponderal (Santana \& Oliveira, 2014).

Dessa forma, conhecer os aspectos que envolvem a adesão dos indivíduos operados aos diversos seguimentos promove subsídios para o desenvolvimento e realização de intervenções eficientes e a falta de conhecimento sobre a taxa de adesão ao seguimento pós-cirurgia bariátrica se torna um complicador quanto ao próprio sucesso da intervenção.

\section{Considerações finais}

Diante dos dados apresentados, e apesar dos diversos e extensos estudos que contemplam a adesão aos cuidados pós-operatórios, destaca-se a necessidade de mais estudos que investiguem a adesão, em seus amplos aspectos. Tais estudos propiciariam ao paciente e à sua rede de apoio maior entendimento da escolha e das consequências.

Há a urgência do desenvolvimento de pesquisas, especialmente na psicologia, direcionadas aos pacientes de cirurgia bariátrica. Muito se discute, mas pouco se sabe sobre os efeitos psicológicos do procedimento cirúrgico em longo prazo. Constructos como a autoeficácia, a resiliência e o suporte social possuem destaque na área da saúde, no tratamento de pacientes em condições crônicas de saúde. Entretanto, são poucos os trabalhos realizados com pacientes submetidos à cirurgia bariátrica.

A avaliação da adesão constitui-se um aspecto complexo, para todos os envolvidos e merece ser estudada mais amplamente. Julga-se serem necessárias mais investigações nesta área, de modo a obter uma melhor compreensão de todo o processo, principalmente no que concerne à exploração do papel que as expectativas parecem exercer em todo o processo e da sua influência sobre a adesão terapêutica dos sujeitos.

\section{Referências}


American Psychological Association. (2012). Manual de publicação da American Psychological Association (6a ed., D. Bueno, trad.). Porto Alegre, RS: Penso.

Abreu-Rodrigues, M. (2014). Apoio social e reganho de peso pós-cirurgia bariátrica: efeitos de intervenção comportamental com cuidadores. (Tese de Doutorado). Universidade de Brasília, Brasília, DF, Brasil.

Andriolli, C., Kuntz, M. G. F., Meurer, V. \& Gonçalves, A. N. (2017). Avaliação da redução de excesso de peso e de carências nutricionais em pacientes pré e pós-cirurgia bariátrica. Revista Brasileira de Obesidade, Nutrição e Emagrecimento. São Paulo. Suplementar 2 v.11. n.68. p.738-747. Jan./Dez.

Barros, L. M., Frota, N. M., Moreira, R. A. N., Brandão, M. G. S. A. \& Caetano, J. A. (2018). Mudanças de hábitos de vida de pacientes em pós-operatório da cirurgia bariátrica. Revista Brasileira de Obesidade, Nutrição e Emagrecimento. São Paulo. v.12. n.74. p.812-819.

Belo, G. Q. M. B., Siqueira, L. T. de, Melo Filho, D. A. A., Kreimer, F., Ramos, V. P \& Ferraz, A. A. B. (2018). Fatores preditivos da perda de seguimento de pacientes submetidos à cirurgia bariátrica. Revista Coluna Brasileira de Cirurgia. 45(2):e 1779. DOI:10.1590/0100-6991e-20181779

Bruziguessi, S. (2010). Adesão ao tratamento multidisciplinar 6 meses após cirurgia bariátrica: influência de fatores socioeconômicos, auto eficácia, suporte social, família e personalidade do paciente. (Dissertação de Mestrado). Pontifícia Universidade Católica de Goiás, Departamento de Psicologia, Goiânia, GO, Brasil.

Camargo-Borges, C. e Japur, M. (2008) Sobre a (não) adesão ao tratamento: ampliando sentidos do autocuidado. Texto contexto - Enferm., vol.17, n.1, pp.64-71.

Cândido, C. F. G. (2015). Associação entre fatores psicossociais e aderência ao tratamento de pacientes submetidos ao tratamento cirúrgico da obesidade. (Dissertação de Mestrado). Faculdade de Juiz de Fora, Minas Gerais, MG, Brasil.

Castanha, C. R., Ferraz, A. A. B., Castanha, A. R., Belo, G. Q. M. B., Lacerda, R. M. R. \& Vilar, L., (2018). Avaliação da qualidade de vida, perda de peso e comorbidades de pacientes submetidos à cirurgia bariátrica. Revista Coluna Brasileira de Cirurgia. 45(3):e 1864. DOI: 10.1590/0100-6991e-20181864

Eduardo, C. A., Silva, A. A., Viana, G C. P., Cruz, C. D. da \& Rocha, R. L. P. (2017). Cirurgia bariátrica: a percepção do paciente frente ao impacto físico, psicológico e social. Revista de Enfermagem do Centro-Oeste Mineiro. 7:e1173. https://doi.org/10.19175/recom.v7i0.1173

Ehrenbrink, P. P, Pinto E. E. P \& Prando F. L. (2009). Um novo olhar sobre a cirurgia bariátrica e os transtornos alimentares. Revista de Psicologia Hospitalar. 7(1); 88-105. 
Fagundes, M. A. B. G., Caregnato, R. C. A. \& Silveira, L. M. de O. (2016). Variáveis psicológicas associadas à cirurgia bariátrica. Aletheia v.49, n.2, p.47-54, jul./dez.

Fandiño J, Benchimol A, Cotinho W, Appolinário J. (2004). Cirurgia Bariátrica: aspectos clínicos-cirúrgicos e psiquiátricos. Revista de Psiquiatria do Rio Grande do Sul.; 26(1):47-51.

Fernandez, R. D., Gomes, D. L., Sá, N. N. B. de, Marcuartú, A. C \& Sousa, I. S. de. (2017). A importância do acompanhamento nutricional em pacientes que realizaram cirurgia bariátrica: relato de experiência. (Anais do VI Congresso de Educação em Saúde da Amazônia (COESA)), Universidade Federal do Pará, Belém, PA, Brasil.

Ferreira, A. B. H. (1999). Novo Dicionário da Língua Portuguesa. Rio de Janeiro: Nova Fronteira.

Garrido, A. B. Jr.. (2004). Cirurgia da Obesidade. São Paulo: Atheneu.

Guerra, L. S. D. (2014). Frequência de transtornos mentais em pacientes obesos candidatos à cirurgia bariátrica por meio de Entrevista Clínica Estruturada para Transtornos do DSM (SCID-I/P) (Dissertação de Mestrado). Faculdade de Medicina, Universidade de São Paulo, São Paulo, SP, Brasil.

Gusmão J.L, Mion Jr. D. (2006). Adesão ao Tratamento- conceitos. Revista Brasileira de Hipertensão vol.13(1): 23-25.

Lima, L. F. de (2012). Cirurgia bariátrica e melhora na qualidade de vida: Revisão integrativa. (Trabalho de conclusão de curso de especialista). Faculdade de Palhoça, Santa Catarina, SC, Brasil.

Lucena M.C., Hazboun A.M., Martins R.R. \& Alchieri J.C. (2010). Aspectos da aderência após cirurgia bariátrica. Revista de Enfermagem UFPE. abr./jun.;4(2):548-56

Marcelino, F. C. (2014). Avaliação das práticas de atividade física em pacientes obesos mórbidos submetidos à operação de by-pass gástrico com reconstrução em Y de Roux com anel. Revista Brasileira de Obesidade, Nutrição e Emagrecimento. São Paulo. $\mathrm{Jan} / \mathrm{Fev}$. v.8. n.43. p.24-30.

Marchesini, S. D. (2010). Acompanhamento psicológico tardio em pacientes submetidos à cirurgia bariátrica. ABCD Arquivos Brasileiros de Cirurgia Digestiva, 23(2), 10813.

Medeiros, A. C. S. (2017). Cirurgia bariátrica no pós-operatório tardio e a adesão ao tratamento nutricional. (Trabalho de conclusão de curso de bacharelado em nutrição). Faculdade de Ciências da Educação e Saúde, UniCEUB, Brasília, DF, Brasil.

Menegotto, A. L. S., Cruz, M. R. R., Soares, F. L., Nunes, M. G. J. \& Branco-Filho, A. J. (2013) Avaliação da frequência em consultas nutricionais dos pacientes após cirurgia bariátrica. Arquivo Brasileiro de Cirurgia Digestiva, 26(2), 117-119. 
Ministério da Saúde (2018). Recuperado de https://www.saude.gov.br/noticias/agenciasaude/45612-brasileiros-atingem-maior-indice-de-obesidade-nos-ultimos-trezeanos\#: : text=Apesar $\% 20 \mathrm{de} \% 20 \mathrm{o} \% 20$ excesso $\% 20 \mathrm{de}$, homens $\% 2 \mathrm{C} 18 \% 2 \mathrm{C} 7 \% 25 . \&$ tex $\mathrm{t}=\mathrm{O} \% 20$ Vigitel $\% 20$ tamb $\% \mathrm{C} 3 \% \mathrm{~A} 9 \mathrm{~m} \% 20$ registrou $\% 20$ crescimento,peso $\% 20$ entre $\% 2$ 0a\%20popula $\%$ C3\%A7\%C3\%A3o\%20brasileira.

Mota D. C. L. (2012). Comportamento alimentar, ansiedade, depressão e imagem corporal em mulheres submetidas à cirurgia bariátrica. (Tese de Mestrado). Faculdade de Filosofia, Ciências e Letras de Ribeirão Preto, São Paulo, SP, Brasil.

Oliveira, D. de A. (2014). Qualidade e comportamento alimentar de mulheres após 24 meses ou mais de cirurgia bariátrica. (Dissertação de pós-graduação). Universidade de Brasília, Brasília, DF, Brasil.

OMS. Organização Mundial da Saúde. (2003). Adherence to long-term therapiesevidence for action. Geneva. Disponível: $<$ http://www.who.int/chp/knowledge/publications/adherence_report/en/>.

OMS. Organização Mundial da Saúde. (2016).

Rocha, A. C., Hociko, K. dos R. \& Oliveira, T. V. de. (2018). Comportamento e hábitos alimentares dos pacientes pós-cirurgia bariátrica. Contextos da Alimentação-Revista de Comportamento, Cultura e Sociedade. Vol. 6 no. 1. São Paulo: Centro Universitário.

Santana, J. T. de \& Oliveira, J. D. de (2014). A importância do acompanhamento nutricional e psicológico no pré e pós-operatório de cirurgia bariátrica. (Trabalho de conclusão de curso de bacharelado em nutrição). Universidade Tiradentes, Aracaju, SE, Brasil.

Santos, M. M. M., Orth, L. C., Prá, M., Ubeti, M. F. \& Trevisol, F. S. (2018). Avaliação da condição de saúde e da qualidade de vida no pós-operatório tardio de pacientes submetidos à cirurgia bariátrica. Revista Brasileira de Obesidade, Nutrição e da qualidade de vida e Emagrecimento. São Paulo. v.12. n.74. p.730-737. Nov./Dez. 2018.

Scabim, V. M., (2011). Adesão ao seguimento nutricional ambulatorial pós operatório de cirurgia bariátrica no Hospital das Clínicas da Faculdade de medicina da Universidade de São Paulo. (Dissertação de Mestrado). Universidade de São Paulo, São Paulo, SP, Brasil.

Schakarowski, F. B., Padoin, A. V., Mottin, C. C. \& Castro, E. K. (2018). Percepção de Risco da Cirurgia Bariátrica em Pacientes com Diferentes Comorbidades Associadas à Obesidade. Temas em Psicologia. Vol. 26, nº 339-346. DOI: 10.9788/TP2018.1-13.

Soares, J. M., Micheletti, J., Oliveira, M. L. de, Silva, A. C. G. da \& Cavagnari, M. A. V. (2017). Práticas alimentares de pacientes em pós-operatório de cirurgia bariátrica: revisão integrativa. BRASPEN J.; 32 (3): 282-7. 
Sociedade Brasileira de Cirurgia Bariátrica e Metabólica. (2018). Consenso Bariátrico Brasileiro. Recuperado de http://www.sbcbm.org.br/associados.asp?menu=2

Teixeira, F. e Maia, A. (2011). Factores preditores do insucesso na gastrobandoplastia: Uma revisão da literatura. Psicologia, Saúde e Doenças, 12 (2), 212-223.

Recebido em: 06/04/2020

Aprovado em: 02/09/2020 